\title{
Gastrointestinal stromal tumor after tyrosine kinase inhibition therapy: a review of biopsies of 34 patients with clinically suspected relapse and/or progression of the tumor
}

\author{
L. PLANK ${ }^{1,2,3, *}$, V. BUZALKOVA ${ }^{3}$, P. SZEPE ${ }^{1,3}$, Z. LASABOVA ${ }^{2,4}$, K. JASEK ${ }^{2,4}$, A. STANCLOVA ${ }^{3,4}$, G. MINARIK ${ }^{5,6}$, J. SUFLIARSKY ${ }^{7}$
}

${ }^{1}$ Martin's Biopsy Center Ltd., Martin, Slovakia; ${ }^{2}$ Division of Oncology, Biomedical Center Martin, Comenius University in Bratislava, Jessenius Faculty of Medicine in Martin, Slovakia; ${ }^{3}$ Department of Pathology, Comenius University in Bratislava, Jessenius Faculty of Medicine and University Hospital in Martin, Slovakia; ${ }^{4}$ Department of Molecular Biology, Comenius University in Bratislava, Jessenius Faculty of Medicine in Martin, Slovakia; ${ }^{5}$ Geneton Ltd., Bratislava, Slovakia; ${ }^{6}$ Department of Molecular Biology, Comenius University in Bratislava, Faculty of Natural Sciences in Bratislava, Slovakia; ${ }^{7}$ Department of Oncology, Comenius University Medical Faculty and National Cancer Institute, Bratislava, Slovakia

${ }^{*}$ Correspondence: plank@jfmed.uniba.sk

Received August 29, 2016 / Accepted December 19, 2016

\begin{abstract}
Implementation of combined surgical and targeted therapy strategies using tyrosine kinase inhibitors improved the prognosis of patients with aggressive GISTs. The therapeutic answer may be individually different, some patients do not respond properly, or even progress in spite of the therapy. This together with intratumoral heterogeneity and possible development of secondary phenotypical and genetical changes represents a challenge for pathologists examining a biopsy of relapsed tumors and/or their metastases. For this study biopsy files of the national Slovak GIST registry were reviewed to identify patients examined bioptically both prior the therapy and during the TKI treatment due to suspected tumor relapse and/or progression. All the GIST biopsies were analyzed using a standardized algorithm of histological, immunohistochemical and molecular analyses of exon 7, 9, 11, 13 of c-KIT and exons 12,14, and 18 of PDGFRA genes, with the aim to identify posttherapeutical changes of these parameters. From 34 patients fulfilling the criteria of selection, all were histologically examined during their clinically suspicious first GIST relaps, eight during the 2nd, three during 3rd and one during 4th and 5th relapse resp. All but one posttherapeutical biopsies showed „viable“ GIST tissue and so 44 relapses of 33 patients could be evaluated in comparison with identical parameters of diagnostic biopsies. Distinguishing three major histological types (spindle-, epitheloid-cell and mixed cell type), a change of the GIST type was identified in $1 / 3$ of 1 st relapse and $1 / 4$ of all relapse biopsies. Evaluation of three phenotypical GIST parameters CD117, CD34 and DOG-1, showed that phenotype alteration was always represented by a single change. The most common was either a gain or loss of CD34 positivity appearing in 1/3 of 1st relapse biopsies, while a loss of CD 117 positivity was identified in one patient's biopsy only. Altogether, the phenotypical changes were in $1 / 4$ of all relapses. A changed mutational profile was recognized in 38,2\% first relaps biopsies and in $33 \%$ of all relapses, the change was mostly isolated (in 10/45 relapses) and less often (in 4/45 relapses) it represented a gain of a new mutation in association with persisting original one. In conclusion, the biopsies of patients showing relapse and/or progression on TKI treatment show predominance of viable GIST cells with limited or even absent signs of scaring, as well as relatively low incidence of morphological, pheno- and genotypical changes.
\end{abstract}

Key words: gastrointestinal stromal tumor, c-KIT mutations, PDGFRA mutations, tyrosine kinase inhibition, targeted therapy, postherapeutical changes

Gastrointestinal stromal tumors (GISTs) are the most frequent mesenchymal malignancies of the gastrointestinal (GI) tract, which develop primarily along the tract and often spread but almost exclusivelly within the abdomen $[1,2]$. They are linked by sharing a common presumed cell of origin, as well as common histological and phenotypical patterns. Although biologically they form a collection of heterogenous molecular entities, more than $80-90 \%$ of GISTs are driven by a pathogenic mutation of either KIT or PDGFRA gene and the others, referred to as wild-type GISTs, harbor mutations of other genes, as e.g. of $S D H, B-R A F$, or $K-R A S$ [3]. Due to ineffectivity of conventional chemotherapy and radiotherapy, for many years 
the only rational therapeutic option for GIST patients was surgery alone. However, although most GISTs are localized at presentation, approximatelly $40 \%$ recur despite macroscopically complete resection [4]. Recently, the understanding of GIST molecular pathogenesis allowed implementation of a biological therapy using tyrosine kinase inhibitors (TKI) targeted to block the effect of driver' mutations. Nowadays, this treatment is used in the clinical practice in adjuvant or neoadjuvant settings in association with surgery [5-9]. The treatment indication is based on assessment of the tumor agressiveness and detection of its molecular pathogenesis $[4,10]$.

Following this challenge, a centralized biopsy diagnostic program for Slovak GIST patients including implementation of molecular analyses was established in 2004; the system is operating due to the cooperation of Slovak pathologists and oncologists. One of the important effects of this cooperation was access to targeted therapy modalities available for GIST patients also in our country based on a precise histopathologi$\mathrm{cal}$ and molecular diagnosis.

At the same time, the implementation of TKI therapy into the clinical practice required a change in the evaluation of therapeutical response. The criteria used for the evaluation of treatment effect assessment in solid tumors (so-called RECIST criteria) based solely on the tumor size changes were insufficient for targeted GIST treatment [11]. The TKI treatment namely leads to the decrease up to „switch-off effect" of metabolic activity of the GIST detectable very early by sensitive positron emission tomography (PET) using fluorine18-fluorodeoxyglucose. Later on, the treatment effect can be assessed by using radiological imaging techniques incl. computed tomography (CT) and others for evaluation of even small previously unrecognizable alterations of the tumor appearance. The imaging techniques have shown that a positive response of GIST after TKI therapy must not necessarily lead to a substantial reduction or shrinkage of tumor mass, but it might be represented by minimal changes or even by a tumor enlargement $[1,11]$. That was the reason to employ quantitative parameters such as small tumor size and tumor density changes into recently defined Choi response criteria [2].

Although most patients with advanced GIST benefit from the treatment, many patients subsequently develop resistance to the applied agent. In many cases the acquired resistance is reported to occur due to a development of secondary mutation in the KIT or PDGFRA genes [7, 12]. In addition, many patients with recurrence and/or metastatic dissemination after TKI treatment may manifest morphological and phenotypical tumor changes $[1,6,11]$. However, in contrast to increasing knowledge on radiological changes only limited data on the GIST morphological changes after TKI therapy verified by tumor biopsy are available. The reported common treatment histological changes include stromal changes and hyalinization due to scarring, the hyalinized areas may contain islands of „surviving" viable tumor cells [7]. The published data sometimes admix radiologic with histopathologic GIST "morphology" changes, or histomorphology after neoadju- vant TKI therapy enabling the tumor resection of primarily unoperable tumor with that of GIST after succesfull and/or failed adjuvant therapy. That was the reason to review the data from our registry with focus on GIST patients showing clinically relapse and/or progression of the disease during the TKI treatment verified by a subsequent biopsy.

\section{Patients and methods}

Patients. The files from registry of Slovak patients with GISTs verified in our center in the period 2004-2015 were reviewed for the data of patients with recorded rebiopsy of either metastasis and or relaps/progression of the primary GIST after targeted TKIs therapy. In all of these patients both primary and all other tumor rebiopsies were represented by tumor tissue fixed in formalin and embedded in paraffin. The biopsies were in the actual time of diagnosis examined first histologically and immunohistochemically (IHC) using a standardized algorithm of examinations. After the verification of the GIST diagnosis the paraffin sections were used for molecular analyses.

Histological and immunohistochemical examinations. Histological examinations using HE, Giemsa and PAS staining and impregnation sec. Gömöri included verification of the diagnosis and determination of the basic histological type of the tumors distinguishing spindle-cell, epitheloid-cell and mixed-cell type respectively. The diagnosis as well as GIST grading and staging were confirmed by at least of one of qualified pathologists (LP and PS). The grading and staging followed accepted criteria [13, 14]; recently, after 2010, also criteria of WHO classification of the GI tract tumors [15].

In addition, occurrence of regressive changes within the neoplastic infiltrates was evaluated and recorded in all the biopsies; these included presence of coagulative necrosis, fibrotisation, fibrohyalinization, of myxoid/cystic myxoid degenerative changes and/or of haemorrhage. For the evaluation, we used a simplified grading system, similar to that of Dennis and Damjanov [7] distinguishing changes appearing in less than $10 \%$ and in more than $10 \%$ of GIST tissue surface area respectively. The evaluation was semiquantitative and was based on data of two observers.

For determination of the GIST phenotype an algorithmical approach including at least detection of CD117, CD34, and since 2011 also of DOG1 was used. In majority of cases when suitable sufficient tissue was available, the panel was supplemented by detection of other antigens contributing to the diagnosis and/or differential diagnosis, as e.g.: muscle-specific actin and smooth muscle actin, desmin, h-caldesmon, S-100 protein, ALK-1, MDM2, CKD-4, SDHB, cytokeratin filaments, Ki-67, etc. Deparaffinization, rehydration, and target retrieval with the Target Retrieval Solution High $\mathrm{pH}(\mathrm{pH}=$ 9) or EDTA $(\mathrm{pH}=9)$ at $96^{\circ} \mathrm{C}$ was performed in the PT Link (Dako PT100). Slides were then processed on the Autostainer Link 48 (Dako AS480) using an automated staining protocol validated for the individual antibodies or mechanically using 
kit EnVision FLEX, High pH, Link in agreement with the producer recommendations. Reagents utilized in addition to mentioned components included a FLEX antibody diluent and FLEX wash buffer, and a hematoxylin counterstain (Dako Denmark A/S, Glostrup, Denmark). IHC-stained slides were mounted in nonaqueous, permanent mounting media.

Molecular analyses. Paraffin-embedded sections were deparaffinized in xylene and washed in descending gradient of ethanol. Genomic DNA was extracted using DNeasy Blood and Tissue Kit (Qiagen, Hilden, Germany) according to the manufacturer's protocol. Detection of mutations located in KIT and PDGFRA exons 9, 11, 13, 17 and 12, 14,18 respectively, was performed as previously published (16). Briefly, using endpoint PCR exons 9, 11, 13 and 17 of KIT and 12, 14 and 18 of PDGFRA were amplified and sequenced by the Sanger method. Additionally to this protocol, samples negative for mutations in exons 9,11,13 and 17 of KIT and 12,14, and 18 of PDGFRA were tested for the presence of mutations in exon 8 of KIT using primers Ex8KIT-F 5'-TTTCCAGCACTCTGACATATGGC-3' and Ex8KIT_R 5 '-TCCCCTCTGCATTATAAGCAGTGC-3'. The PCR was performed with $50 \mathrm{ng}$ of DNA in $25 \mu \mathrm{l}$ reaction mixture containing $25 \mathrm{nM}$ of each forward and reverse primer, $250 \mu \mathrm{M}$ of each of the four dNTPs (Gene Amp dNTP Mix with dTTP, Thermo Fisher Scientific, USA), $2.5 \mathrm{mM} \mathrm{MgCl}_{2} 2.5 \mu \mathrm{l}$ of 10xPCR buffer and 1 Unit of FastStart Taq DNA Polymerase (Roche Diagnostics, Germany). Thermal cycling condition included $10 \mathrm{~min}$ at $95^{\circ} \mathrm{C}$, followed by 30 cycles of $94^{\circ} \mathrm{C}$ for $30 \mathrm{~s}, 67^{\circ} \mathrm{C}$ for $1 \mathrm{~min}$ and $72{ }^{\circ} \mathrm{C}$ for $1 \mathrm{~min}$ and one cycle of $72{ }^{\circ} \mathrm{C}$ for $5 \mathrm{~min}$. The PCR products were analyzed by agarose electrophoresis, purified by NucleoSpin Gel and PCR Cleanup Kit (Macherey\&Nagel, Germany) and sequenced with the BigDye Terminator v1.1 Cycle Sequencing Kit (Thermo Fisher Scientific, USA) according to the manufacturer's protocol on 3500 Genetic Analyzer (Applied Biosystems, USA). The sequences were analysed using the SeqScape software v2.7 (Applied Biosystems, USA).

Processing of the data. For the evaluation, patient's data of the first diagnostic biopsy were compared with those of 1st relaps rebiopsy; in cases with history of multiple relapses always with those of previous biopsy. The evaluation included: a) anatomical localisation of tumors, the GIST was considered to represent an extragastrointestinal GIST („e-GIST“) when a direct anatomical association with any of the luminal GI organs could not be proved, neither by surgeon nor by pathologist, b) GIST histomorphological typing, c) changes of CD117, CD34 (and DOG1) IHC expressions, d) mutation status of KIT and PDGFRA genes resp., and e) presence and extent of regressive changes as described above.

\section{Results}

Patients and their biopsies. Altogether data of 34 patients fulfilling the required criteria were identified in our files. The series consisted of 20 male (58.8\%) and 14 female (41.2\%)
GIST patients, their age at the time of primary diagnosis was within the interval $22-89$ years, with median of 56.3 years. In all of the patients, the rebiopsies were indicated due to clinically suspicious relapse and/or GIST progression after TKI treatment. The tissue was obtained by different methods including partial or total surgical tumor and/or metastasis resection, core and endoscopic biopsy, etc. From 34 patients of the series, all were histologically examined during their clinically suspicious first GIST relaps, 8 during the second, 3 during third and one of them also during his 4th and 5th relapse. In majority of the patients, all parameters of primary GISTs were verified in our registry. In a limited number of patients (see further), some data had to be obtained from the patient's documentation for different reasons (primary patient's operation in abroad, or the primary tumor was not registered and this tissue was no more available).

The localization of primary and relapsed tumors is summarized in Table 1 . The most common primary GISTs were small intestinal tumors $(17 / 34=50 \%)$, followed by gastric $(10 / 34=$ 29.4\%), (intra-) abdominal soft tissue (AST) E-GISTs (5/34 = $14.7 \%)$ and large intestinal GIST $(2 / 34=5.9 \%)$. The clinically suspicious 1st recidive's tissue of 32/34 patients was obtained from one of following localizations: local relaps $(5 / 34=14.7 \%)$, relaps in other GI organ $(4 / 34=11.8 \%)$, GIST metastasis to AST, either single or multiple (7 and 10 patients, resp., e.g. together $17 / 34=50 \%)$, metastasis to liver $(6 / 34=17.6 \%)$ and urinary bladder $(1 / 34=2.9 \%)$. In 2 of 34 patients $(5.8 \%)$ the biopsy of the 1st relaps included examinations of two different localizations: local recidive together with AST metastasis and coincidence of metastasis to liver and AST, respectively. The relaps of the disease was histologically confirmed in 33/34 patients (97.1\%). Biopsy of the first relapse of one patient with expected AST metastasis consisted of fully hyalinized tissue and contained neither vital and nor necrotic GIST tissue.

Second relaps of GISTs was bioptically examined in $8 / 34$ patients, either in biopsy of solitary and less common multiple metastasis to AST, in relaps appearing in other GI tract organ, and/or to synchronous metastasis to liver and lungs. Third relaps was proved as local recidive in one patient with primary gastric GIST. The second patient with primary small intestinal tumor developed hepatal metastasis during both 1st and 2nd relaps, synchronous liver and AST metastasis in 3rd and again hepatal metastases during 4 th and 5 th recidive.

Histological types of the GISTs. The overview of all results on the GIST type is summarized in Table 2. In summary, the primary GISTs represented following histological types: spindle-cell in 18/34 (52.9\%), epitheloid in 6/34 (17.6\%) and mixed cell GIST type in 10/34 (29.4\%) patients. As already mentioned, the relaps biopsy of one of the patients did not show any GIST tissue. In 22/33 (66.7\%) cases the type of the 1 st relapse was unchanged in the 1st relapse biopsy, this was true for all 6 patients with primary epitheloid-cell type, 13/18 with primary spindle-cell type and for $3 / 10$ patients with primary mixed-cell type. 11/33 patients rebiopsies (33.3\%) showed different GIST type in contrast to the primary one. 
Table 1. Summary of localisation of the primary and relapsing GISTs.

\begin{tabular}{|c|c|c|c|c|c|c|}
\hline \multicolumn{7}{|c|}{ Localisation of } \\
\hline $\begin{array}{l}\text { Primary tumor } \\
(\mathbf{n}=\mathbf{3 4})\end{array}$ & $\begin{array}{l}\text { 1st relaps } \\
(n=34)\end{array}$ & & $\begin{array}{l}\text { 2nd relaps } \\
\qquad(\mathrm{n}=8)\end{array}$ & $\begin{array}{l}\text { 3rd relaps } \\
\quad(\mathbf{n}=2)\end{array}$ & $\begin{array}{l}\text { 4th relaps } \\
\qquad(\mathrm{n}=1)\end{array}$ & $\begin{array}{l}\text { 5th relaps } \\
\qquad(\mathrm{n}=1)\end{array}$ \\
\hline \multirow{6}{*}{$\begin{array}{l}\text { Stomach } \\
(n=10)\end{array}$} & local & 2 & - & 1 & - & - \\
\hline & hepatal MTS & 3 & $1^{* * *}$ & - & - & - \\
\hline & MTS to AST & 3 & - & - & - & - \\
\hline & pulmonary MTS & - & $1^{* * *}$ & - & - & - \\
\hline & large intestine & 1 & - & - & - & - \\
\hline & AST without vital tumor & 1 & & & - & - \\
\hline \multirow{5}{*}{$\begin{array}{l}\text { Small intestine } \\
(n=17)\end{array}$} & local & $3^{*}$ & - & - & - & - \\
\hline & large intestine & 1 & 1 & - & - & - \\
\hline & stomach & 1 & - & - & - & - \\
\hline & hepatal MTS & $3^{\#}$ & $1^{\#}$ & $1^{\#}$ & $1^{*}$ & $1^{*}$ \\
\hline & MTS to AST & $10^{*}$ & 3 & $1^{\#}$ & - & - \\
\hline \multirow{2}{*}{$\begin{array}{l}\text { Large intestine } \\
(\mathrm{n}=2)\end{array}$} & MTS to AST & 1 & 1 & - & - & - \\
\hline & MTS to urinary bladder & 1 & - & - & - & - \\
\hline \multirow{3}{*}{$\begin{array}{l}\text { E-GIST } \\
(\mathbf{n}=5)\end{array}$} & small intestine & 1 & 1 & - & - & - \\
\hline & hepatal MTS & $1^{\star *}$ & - & - & - & - \\
\hline & MTS to AST & $4^{\star *}$ & - & - & - & - \\
\hline
\end{tabular}

For abbreviations.: AST abdominal (intraabdominal) soft tissue, e.g. omentum, peritoneum, retroperitoneum, etc.

* in 1 patient the 1st relaps identified both in small intestine and AST MTS; ${ }^{* *}$ in 1 patient the 1 st relaps identified both in hepatal and AST MTS; *** in 1 patient the 2nd relaps identified both in hepatal and pulmonary MTS; \# in 1 patient the 1st and 2nd relaps identified in the hepatal AST, the 3rd both in hepatal and AST MTS.

This was common in 7/18 (38.9\%) patients with primary mixed-cell type GISTs: 4 patients showed change to spindle-, 2 to epitheloid-cell type, and the last patient showed epitheloid morphology in local small intestinal recidive and spindle-cell type in synchronous metastasis to AST. Biopsies of 1 st relaps of 5/18 (27.8\%) primary spindle-cell type demonstrated change to epitheloid- in 4 and to mixed-cell type in 1 patient.
The examinations of 8 patients with bioptically verified $2 \mathrm{nd}$ relapse did not show any type change when comparing 2 nd versus 1st relaps biopsy. The same is true for both the patients with bioptically verified $3 \mathrm{rd}$ and for one patient developing 4th and 5th relapses.

Altogether a change of the GIST histological type occured in 11 of all $45(24.4 \%)$ relapses of analyzed patients recidives in the series.

Table 2. Summary of the histological types of the primary and relapsing GISTs.

\begin{tabular}{|c|c|c|c|c|c|c|c|}
\hline \multirow{2}{*}{$\begin{array}{l}\text { GISTlocalisation } \\
\text { primary tumor } \\
(\mathrm{n}=34)\end{array}$} & \multicolumn{7}{|c|}{ Histological GIST type ${ }^{\star *}$} \\
\hline & $\begin{array}{l}\text { primary tumor } \\
(\mathrm{n}=34)\end{array}$ & & $\begin{array}{c}\text { 1st relaps } \\
(\mathbf{n}=34)\end{array}$ & $\begin{array}{l}\text { 2nd relaps } \\
\qquad(\mathrm{n}=8)\end{array}$ & $\begin{array}{c}\text { 3rd relaps } \\
\quad(n=2)\end{array}$ & $\begin{array}{l}\text { 4th relaps } \\
\quad(n=1)\end{array}$ & $\begin{array}{l}\text { 5th relaps } \\
(\mathbf{n}=1)\end{array}$ \\
\hline \multirow{4}{*}{$\begin{array}{l}\text { Stomach } \\
(n=10)\end{array}$} & Spindle cell type & 4 & 2 & - & - & - & - \\
\hline & Epitheloid cell type & 4 & 7 & 1 & 1 & - & - \\
\hline & Mixed cell type & 2 & - & - & - & - & - \\
\hline & Without vital tumor tissue & - & 1 & - & - & - & - \\
\hline \multirow{3}{*}{$\begin{array}{l}\text { Small intestine } \\
(\mathrm{n}=17)\end{array}$} & Spindle-cell type & 8 & $12^{*}$ & 4 & 1 & 1 & 1 \\
\hline & Epitheloid cell type & 1 & $3^{*}$ & 1 & - & - & - \\
\hline & Mixed & $\star 8$ & 3 & - & - & - & - \\
\hline \multirow{3}{*}{$\begin{array}{l}\text { Large intestine } \\
(n=2)\end{array}$} & Spindle-cell type & 2 & 2 & 1 & - & - & - \\
\hline & Epitheloid cell type & - & - & - & - & - & - \\
\hline & Mixed cell type & - & - & - & - & - & - \\
\hline \multirow{3}{*}{ E-GIST $(n=5)$} & Spindle-cell type & 4 & 1 & 1 & - & - & - \\
\hline & Epitheloid cell type & 1 & 2 & - & - & - & - \\
\hline & Mixed cell type & - & 1 & - & - & - & - \\
\hline
\end{tabular}


Immunohistochemically defined phenotypes of GISTs. Evaluation of phenotypical patterns was focused on expression of two throughout the whole analyzed period standardly used basic GIST markers CD117 and CD34, the results are summarized in Table 3. Altogether, the primary GISTs showed positive CD117 expression in biopsies of 31/33 (93.9\%) and CD34 positivity in $26 / 34$ patients (76.4\%), while the phenotype of primary GIST of 1 patient remained unknown. The phenotype remained unchanged in relaps biopsies of 26/33 (78.8\%) patients, in $7 / 33(21.2 \%)$ patients following single phenotypical alterations occurred: loss of CD34 positivity in 2/33 (6.1\%) patients, loss of CD117 positivity in $1 / 33(3.0 \%)$ and gain of CD34 positivity in biopsies of 4/33 (12.1\%) patients. However, in one of the last mentioned cases, the gain of $\mathrm{CD} 34$ positivity was present in biopsy taken from local duodenal recidive only, while a synchronous metastasis to omentum was CD34 negative (as in the primary biopsy). One patient, as already mentioned, did not show any tumor tissue in the rebiopsy excision of AST.

Evaluation of the second relapse biopsies of 5/8 (62.5\%) patients showed unchanged phenotype when compared with that of primary tumor. In one patient a loss and in one a gain of $\mathrm{CD} 34$ expression respectively were identified, and in the last patient the phenotype was unknown. Evaluation of the 3rd relaps biopsies identified one patient with a gain of CD34 positivity, while in the second patient's the relapsed GIST showed unchanged phenotype) and the same was true for his 4 th and 5th relaps. Altogether the change of either CD117 and/or CD34 IHC expression occured in 11 of all $45(24.4 \%)$ relapses of analyzed patients recidives in the series.

A positive DOG1 expression was verified in primary tumors of three patients and in relaps biopsies of 10 patients, the
DOG1 positive expression remained unchanged in all biopsies with exception of one case with two synchronous localizations of 1st relaps: the local duodenal recidive showed (in addition to the gain of CD34 positivity) a loss of DOG1 positivity, while a metastasis to the omentum (losing CD34 expression - see above) was DOG1 positive.

Neither primary nor relapsed biopsies showed positivity of other antigens used for the differential diagnosis and/or discrimination of other tumors from the GISTs, e.g. ALK1, MDM2, CDK4, cytokeratin filaments etc.

Molecular analyses of GISTs. The results of are summarized in Table 4 . The analyses of the primary GISTs verified: KIT exon 11 mutations in 15 patients, KIT exon 9 in 5 patients, PDGFRA exon 18 mutations (c.252A-T, pD842V) in 2 patients, WT genotype in 9 patients, while in 3 patients the mutational status remained unknown.

DNA analyses obtained from 1st relaps biopsies showed unchanged mutational status of 21/34 (61.8\%) patient's tumors. In 10/34 (29.4\%) patients the genotype was changed as follows: loss of original mutation in 5 patients, gain of a second mutation in association with persisting primary in 3 patients, new mutation in primary WT GIST in 1 patient, and mutation in 1 patient with originally unknown status. The results of 1st relaps DNA analysis of 2 patients were not accessible and in relaps biopsy of one patient vital GIST tissue was not present. Mutation status identified in 2nd relaps compared with that of 1st one was following: loss of mutation in one patient, unchanged type of mutation in 5 patients, gain of a new mutation of exon 17 in association with persisting primary exon 11 mutation of KIT in one, and unknown status in one patient. The mutation status of one patient in the 3rd relaps was unknown, the second patient with previously unknown status showed WT/WT genotype in the 3rd relaps and gained

Table 3. Summary of CD117 and CD34 expressions of the primary and relapsing GISTs.

\begin{tabular}{|c|c|c|c|c|c|c|}
\hline \multicolumn{7}{|c|}{$\begin{array}{l}\text { CD117 and CD34 phenotype detected in biopsy of } \\
\text { ( } n=\text { number of patients) }\end{array}$} \\
\hline $\begin{array}{l}\text { primary tumor } \\
(\mathrm{n}=34)\end{array}$ & $\begin{array}{l}\text { 1st relaps } \\
(\mathbf{n}=34)\end{array}$ & & $\begin{array}{l}\text { 2nd relaps } \\
\qquad(\mathrm{n}=8)\end{array}$ & $\begin{array}{l}\text { 3rd relaps } \\
\quad(\mathrm{n}=2)\end{array}$ & $\begin{array}{l}\text { 4th relaps } \\
\quad(\mathrm{n}=1)\end{array}$ & $\begin{array}{l}5 \text { th relaps } \\
\quad(\mathrm{n}=1)\end{array}$ \\
\hline \multirow{4}{*}{$\begin{array}{l}\text { CD117+ / CD34+ } \\
(\mathrm{n}=24)\end{array}$} & unchanged & 21 & 4 & 1 & 1 & 1 \\
\hline & loss of CD34 positivity & 2 & 1 & - & - & - \\
\hline & gain of CD34 positivity & - & & $1^{\#}$ & & \\
\hline & without vital tumor tissue & 1 & - & - & - & - \\
\hline \multirow{3}{*}{$\begin{array}{l}\text { CD117+ / CD34- } \\
(\mathrm{n}=7)\end{array}$} & unchanged & $3^{*}$ & - & - & - & - \\
\hline & gain of CD34 positivity & $4^{*}$ & 1 & - & - & - \\
\hline & loss of CD117 positivity & 1 & - & - & - & - \\
\hline CD117- / CD34+ $(n=1)$ & unchanged & 1 & 1 & - & - & - \\
\hline CD117- / CD34- $(\mathrm{n}=1)$ & unchanged & 1 & - & - & - & - \\
\hline CD117+ / CD34 U/ND (n=0) & & $1^{\#}$ & & - & - & - \\
\hline $\mathrm{U} / \mathrm{ND}^{*}(\mathrm{n}=1)$ & & - & $1^{\#}$ & - & - & - \\
\hline
\end{tabular}


Table 4. Summary of molecular changes of the primary and relapsing GISTs.

\begin{tabular}{|c|c|c|c|c|c|c|c|c|c|}
\hline \multicolumn{10}{|c|}{ Localisation and type of the mutation in ( $n=$ number of patients) } \\
\hline \multirow{2}{*}{$\begin{array}{l}\text { primary tumor } \\
\text { gene/exon/type } \\
(n=34)\end{array}$} & & & \multicolumn{2}{|c|}{$\begin{array}{c}\text { 1st relaps } \\
(\mathrm{n}=34)\end{array}$} & \multicolumn{2}{|c|}{$\begin{array}{l}2 \text { nd relaps } \\
\qquad(\mathrm{n}=8)\end{array}$} & \multirow{2}{*}{$\begin{array}{l}\text { 3rd relaps } \\
(\mathrm{n}=2)\end{array}$} & \multirow{2}{*}{ 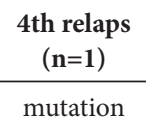 } & \multirow{2}{*}{$\begin{array}{c}\begin{array}{c}5 \text { th relaps } \\
(\mathrm{n}=1)\end{array} \\
\text { mutation }\end{array}$} \\
\hline & & & loss/gain of & identical & loss/gain of & identical & & & \\
\hline \multirow{4}{*}{$\begin{array}{c}K I T / 9 \\
(n=5)\end{array}$} & ins & 4 & $1 /-$ & 2 & $-1-$ & 2 & - & - & - \\
\hline & del & 1 & $1 /-$ & - & $-1-$ & - & - & - & - \\
\hline & PM & - & $-/-$ & - & $-/-$ & - & - & - & - \\
\hline & other & - & $\begin{array}{c}-/ 1 \\
\text { del11 } \\
\end{array}$ & - & $-/-$ & - & - & - & - \\
\hline \multirow{4}{*}{$\begin{array}{c}K I T / 11 \\
(n=15)\end{array}$} & ins & 1 & $-/ 1$ & $2^{*}$ & $-1-$ & - & - & - & - \\
\hline & del & 12 & $1 /-$ & 9 & $-1-$ & $3^{* *}$ & - & - & - \\
\hline & $\mathrm{PM}$ & 2 & $1 / 1^{*}$ & - & $-/-$ & - & - & - & - \\
\hline & other & - & $\begin{array}{c}-/ 1 \\
\text { PM } 9\end{array}$ & - & $-/-$ & $\begin{array}{c}\mathbf{1}^{\star *} \\
\text { PM } 17\end{array}$ & - & - & - \\
\hline \multirow{2}{*}{$\begin{array}{l}\text { PDGFRA / } 18^{\#} \\
(\mathrm{n}=2)\end{array}$} & PM & 2 & $1 /-$ & - & $-1-$ & - & - & - & - \\
\hline & other & - & $-/-$ & $\begin{array}{c}1 \\
\mathrm{U} / \mathrm{ND} \\
\end{array}$ & $-/-$ & - & - & - & - \\
\hline \multirow{2}{*}{$\begin{array}{l}\text { WT } \\
(n-9)\end{array}$} & WT & 9 & $-/-$ & 8 & $-/-$ & 1 & - & - & - \\
\hline & other & - & $\begin{array}{c}-/ 1 \\
\text { del } 11 \\
\end{array}$ & - & $-1-$ & - & - & - & - \\
\hline \multirow{2}{*}{$\begin{array}{l}\mathrm{U} / \mathrm{ND} \\
(\mathrm{n}=3)\end{array}$} & $\mathrm{U} / \mathrm{ND}$ & 3 & $\begin{array}{c}-/ 1 \\
\text { PM } 11\end{array}$ & $\begin{array}{c}1 \\
\mathrm{U} / \mathrm{ND}\end{array}$ & $\begin{array}{c}\text { 1/- } \\
\text { PM11 }\end{array}$ & $\begin{array}{c}1 \\
\mathrm{U} / \mathrm{ND}\end{array}$ & $\begin{array}{c}1 \\
\mathrm{U} / \mathrm{ND}\end{array}$ & - & - \\
\hline & other & - & \multicolumn{2}{|c|}{$\begin{array}{c}\mathbf{1} \\
\text { no vital tissue }\end{array}$} & $-1-$ & - & $\begin{array}{c}1 \\
\mathrm{WT}\end{array}$ & $\begin{array}{c}1 \\
\text { gain of ins } 9\end{array}$ & $\begin{array}{c}1 \\
\text { gain of ins } 9\end{array}$ \\
\hline
\end{tabular}

Abbreviations: del = deletion, $\mathrm{PM}=$ point mutation, ins = insertion, WT $(\mathrm{WT} / \mathrm{WT})=$ without detectable mutation in the examined exons of KIT and PDGFRA resp., $\mathrm{U}=$ unknown, $\mathrm{ND}=$ not done

* one patient showed in the 1st relapse persistence of exon 11 ins and gained second exon 11 point mutation; ${ }^{* *}$ one patients showed persistence of exon 11 del mutation during the 1st and 2nd relaps and gained in addition in the 2 nd relaps second exon 17 point mutation; ${ }^{*}$ both patients with p.D842V mutation

new mutation of exon 9 in the 4th relaps, the same mutation was detected also in the last 5th relaps of this patient.

Altogether the change of mutation' type was detected in our register in 13/34 (38.2\%) patients and 15 of all 45 (33.3\%) patients relapses in the series respectively. In spite of some missing data we can summarize that the change was isolated in $10 / 45(22.2 \%)$ relapses, while in $4 / 45(8.9 \%)$ relapses it represented a gain of a new mutation in association with persisting original mutation.

Regressive changes and fibrosis of GISTs. All the data are summarized in Table 5 . The biopsies of primary tumors showed inextensive areas of focal: a) acute haemorrhage in $15 / 32$, b) myxoid degeneration in $11 / 32$, c) coagulative necrosis (with "ghost" necrotic tumor cells) in $8 / 32$, d) fibrosis in $3 / 32$, e) dystrophic calcification in one tumor, and in two patients the primary tumor tissue was not accessible for the evaluation. In all of these focal lesion, their extent was smaller than $5 \%$ of GIST tissue surface area.

In addition to less frequent identical focal changes in relapse biopsies of many patients (details see in Table 5), the relaps biopsies of some patients showed also more extensive areas (shown in Table 5 with bold type letters) of: a) fibrohyalinisation, either acellular or hypocellular, or with foci (,,islands“) of vital tumor tissue - this change was observed in 11/45 (24.4\%) relaps biopsies (see Figure 1), and of b) myxoid pseudovacuolar and/or cystic myxoid changes in 4/45 (8.9\%) relaps biopsies. With exception of the above mentioned patient showing only fibrohyalinized tissue without any GIST tissue in the first relapse biopsy, these changes in all other biopsies occupied approx. $10-20 \%$ of the GIST surface area. In addition, in some posttreatment biopsies also more-less extensive areas of acute haemorrhage were observed.

\section{Discussion}

Recent advances in understanding the molecular GIST pathogenesis followed by implementation of precise histopathological, phenotypical, and molecular GIST diagnosis have substantially contributed to positive changes of GIST patients outcome. The introduction of centralized program on GIST diagnosis in Slovakia in 2004 has facilitated access to new therapeutic modalities for the patients, allowed a population-based study of GIST mutations [16] and successful inclusion of Slovak GIST Registry data into large European meta-analyses $[4,10]$. In this study pre- and post-treatment biopsies of patients with GIST relapsing after TKI therapy registered in the program were analyzed to identify the changes of the tumor's parameters. 

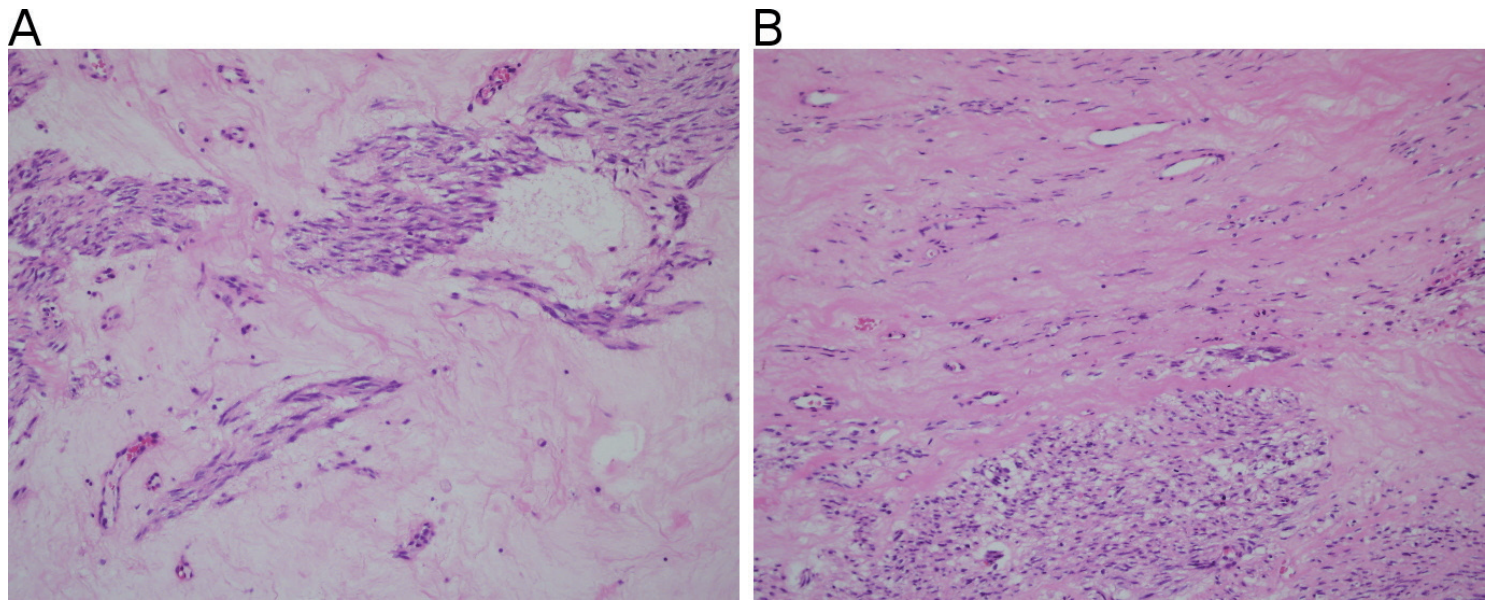

Figure 1. A/ extensive hyalinosis and myxoid regressive changes of GIST after targeted therapy (HE staining, 20x), B/ residual island of „vital“ GIST tissue within areas of fibrohyalinisation (HE staining, 20x)

Table 5. Summary of morphological changes of GIST tissue in primary tumors and their relapses

\begin{tabular}{|c|c|c|c|c|c|c|c|}
\hline Primary GIST localisation & & & Morpho & l (regressiv & anges in & & \\
\hline$(n=34)$ & $\begin{array}{l}\text { primary tumor } \\
(\mathrm{n}=34 \text { patients })\end{array}$ & & $\begin{array}{c}\text { 1st relaps } \\
(\mathrm{n}=34)\end{array}$ & $\begin{array}{l}\text { 2nd relaps } \\
\qquad(\mathrm{n}=8)\end{array}$ & $\begin{array}{l}\text { 3rd relaps } \\
\qquad(\mathrm{n}=2)\end{array}$ & $\begin{array}{l}\text { 4th relaps } \\
\qquad(\mathrm{n}=1)\end{array}$ & $\begin{array}{c}\text { 5th relaps } \\
\quad(n=1)\end{array}$ \\
\hline & haemorrhage & 6 & 2 & - & - & - & - \\
\hline & necrosis & 2 & 2 & - & - & - & - \\
\hline & fibrotisation & 3 & - & - & - & - & - \\
\hline Stomach & myxoid degeneration & 6 & 2 & - & - & - & - \\
\hline & $\mathrm{U} / \mathrm{ND}$ & $2^{*}$ & $1^{*}$ & $1^{*}$ & & & \\
\hline & extensive hyalinisation & - & 5 & - & $1^{*}$ & - & - \\
\hline & extensive myxoid changes & - & - & - & - & - & - \\
\hline & haemorrhage & 7 & 11 & 4 & 1 & 1 & 1 \\
\hline & necrosis & 4 & 5 & 1 & - & - & - \\
\hline Small intestine & fibrotisation & - & 4 & 1 & 1 & 1 & 1 \\
\hline$(n=17)$ & myxoid degeneration & 5 & 7 & 2 & - & - & - \\
\hline & extensive hyalinisation & - & 3 & - & - & - & - \\
\hline & extensive myxoid changes & - & 1 & 2 & 1 & - & - \\
\hline & haemorrhage & - & 1 & - & - & - & - \\
\hline & necrosis & - & - & - & - & - & - \\
\hline & calcification & 1 & - & - & - & - & - \\
\hline Large intestine & fibrotisation & - & - & 1 & - & - & - \\
\hline & myxoid degeneration & - & - & 1 & - & - & - \\
\hline & extensive hyalinisation & - & - & - & - & - & - \\
\hline & extensivemyxoid changes & - & - & - & - & - & - \\
\hline & haemorrhage & 2 & 4 & 1 & - & - & - \\
\hline & necrosis & 2 & 2 & - & - & - & - \\
\hline E-GIST & fibrotisation & - & 1 & 1 & & - & - \\
\hline$(\mathbf{n}=5)$ & myxoid changes & - & 1 & - & - & - & - \\
\hline & extensive hyalinisation & & 2 & - & - & - & - \\
\hline & extensive myxoid changes & - & - & - & - & - & - \\
\hline
\end{tabular}

* Two patients without available primary GIST tissue for evaluation of the analyzed changes, for one of them at least the 3rd relaps biopsy could be evaluated Explanation: The morphological changes described in thick letters appearing in grey boxes represent posttherapeutical changes exceeding more than $10 \%$ of the tumor surface, other are either focal and/or minimal only. 
Generally, GIST patients may be treated by a combination of surgery and targeted therapy - either in neoadjuvant setting of primary unresectable tumor or by a surgical resection associated with adjuvant TKI therapy of patients with aggressive GISTs $[3,11]$. The targeted therapy leads to metabolic and morphological changes of the tumor tissue detectable by radiological examination incl. CT and other imaging techniques, and/or by biopsy examinations $[1,7,11,17-19]$. The desired effects of targeted therapy on the tumor morphology and „size“ might be variable in different settings. A neoadjuvant therapy is expected to reduce a tumor mass allowing its subsequent surgical resection $[6,9]$. However, the metastatic adjuvant therapy must not lead to a substantial decrease of tumor size and therefore the traditional response assessment criteria failed to be sufficiently relevant [20]. Recently, new so called Choi GIST response criteria, based on radiologic evaluations of tumor density and morphology alterations have been developed for a proper therapeutic response evaluation $[1,2,11,17-19]$. They allow to recognize therapy changes such as even small tumor size alterations, or a "stable disease" or a "pseudoprogression“ caused by tumor enlargement, e.g. due to secondary cystic, myxoid, "necrotic" and/or intratumoral haemorrhagic changes $[2,7,17,18,21]$.

Histologically, GISTs after TKI succesful treatment may show decrease of tumor cellularity and stromal alterations including various degrees of sclerosis/hyalinosis and myxoid/ cystic myxoid changes, but an extensive tumor necrosis is not a frequent finding $[7,8]$. In the first patient treated with imatinib, biopsy specimens at 1 and 2 months after starting treatment showed a marked decrease in tumor cells as well as myxoid degeneration and scarring [3]. Since the introduction of TKI treatment, the biopsies representing successfully resected GISTs after neoadjuvant treatment represent a challenge to pathologist to evaluate a residual disease in a background of regressive and healing changes [7]. They include mostly dominating areas of hyalinization and scarring, containing either residual ,island“ of tumor tissue or few or no remaining tumor cells $[5,12,23]$.

However, the radiologic response rates show inadequate correlation with histopathologic alterations and pathologic response rates. In addition, it is to be expected that the findings in patient's biopsy after a good therapeutic response might be different from that of patient showing progression on TKI treatment. All that seems to be relevant for evaluation of our patients, who manifested clinically suspected progression and/ or relaps of the disease on TKI treatment and were therefore rebiopsied. The clinical suspicion of a relaps was histologically proven in all but one patient and in 44 of 45 rebiopsies. The rebiopsy of one single patient, examined at clinically expected first relapse, contained neither histologically nor immunohistochemically any residual tumor cells by finding of hyalinized fibrous tissue only. In contrast, in all other in relapse rebiopsies of 33 from 34 patients in the series, the biopsy specimens contained flourishing tumor tissue with inextensive areas of either haemorrhagic or regressive changes including necrosis, fibrosis etc. Of course we can not fully exclude a sampling error by second opinion reading of the biopsy. However, only in less than $25 \%$ of all rebiopsy examinations we could observe more extensive areas of hyalinization and or myxoid changes, occupying more than $10 \%$ of the tumor surface area inside the dominating „vital“ tumor areas. These findings might be considered to represent histomorphologic expression of the treatment failure, although associated with different clinical manifestations, e.g local relapse or progression, or metastatic dissemination of the disease.

Regardless of intratumoral heterogeneity problems, such treatment failures might be associated with primary and/or secondary developed resistance. The primary resistance to TKI treatment has been reported in approximately $15 \%$ of patients with GIST and the surgical specimens from such patients show a correlating lack of histological response [7]. The proportion of patients with primary GIST mutations known to be associated with inferior outcome and/or recurrence free survival or with suboptimal therapeutic response was relatively high in our series. Five of our patients had KIT exon 9 mutations, two PDGFRA exon 18 substitution mutation leading to Asp842Val (p.D842V) and 9 patients wild-type GISTs lacking KIT and PDGFRA mutations. In addition, other 12 of 15 patients with KIT exon 11 mutation showed its deletion type reported to be associated with high recurrence risk [10]. Altogether at least in 28 of 34 patients the mutation profile of their primary tumor might help to understand the course of their disease. Another clinical problem is the resistance to applied agent based on occurrence of secondary mutation in the KIT or PDGFRA genes $[7,12]$. In the biopsies taken throughout the disease relapse and/or progression, we have identified a changed KIT or PDGFRA mutation type - either loss or gain of previous mutations in approximately $1 / 3$ of our patients and their relapses. However, a secondary mutation acquired in addition to the primary one was observed in 4 of 45 (8.9\%) relapses only. One of the secondary arising mutations was represented by KIT exon 17 mutation being associated with moderate sensitivity to TKI treatment [10].

It has been discussed that a GIST relapse is often associated with an alteration of its phenotypical parameters [7]. Relapsed GISTs in our cohort showed changed histotype in $24.4 \%$ of all relapses only, majority of changes appeared in cases with primary mixed-cell type. In contrast, no change of histological GIST type was observed throughout $75 \%$ of all relapses, including the patients with multiple relapses. In spite of some missing phenotype data of few patients of our series, similar results were proved by our analysis of GIST phenotype, what might allow two conclusions. First, the change of two basic phenotype GIST markers CD117 and CD34 was not as frequent, as it occurred in approx. $25 \%$ of all relapses of our patients. In addition, the phenotype of GIST of limited number of our patients with multiple relapses was relatively stable. Second, the intratumoral heterogeneity is indeed a rare phenomenon, but may lead to different IHC profile of simulta- 
neously appearing relapsing GISTs at different sites [24]. This is true also for the observed loss of DOG1 positivity, which is considered to be the most sensitive of GIST markers.

In summary, it is reasonable to understand that all the morphological and phenotypical changes might have an impact not only on treatment decisions and diagnostic accuracy in relapsing GISTs [7] but also on prognosis of the patients. However, they appeared in the cohort of our patients in approx. 1/4 and a change of KIT or PDGFRA mutation type in approx. 1/3 of relapse biopsies only. For the prognosis of the patients, the intrinsic properties of the tumor, e.g. primary GIST mutations known to be associated with inferior outcome and tumor heterogeneity [24] seem to be of great importance. A rate of secondary mutations of these genes was relatively low, as they were recognized in less than $10 \%$ of all relapses. It seems to be plausible to involve also other than KIT and PDGFRA gene mutational status into the complex diagnostic algorithm of GIST biopsies.

Acknowledgments: Supported by Grant APVV-14-0273, Educational grant of Novartis Oncology, Slovakia and by Center of BioMed Martin (ITMS code 26220220187) at Comenius University in Bratislava, Jessenius Faculty of Medicine in Martin, which are co-financed by EU sources.

\section{References}

[1] HEINICKE T, WARDELMANN E, SAUERBRUCH T, TSCHAMPA HJ, GLASMACHER A et al. Very early detection of response to imatinib mesylate therapy of gastrointestinal stromal tumours using 18Fluoro-Deoxyglucose-Positron emission tomography. Anticancer Res 2005; 25: 4591-4594.

[2] CHOI H. Response evaluation of gastrointestinal stromal tumors. Oncologist 2008; 13 (Suppl. 2): 4-7. https://doi. org/10.1634/theoncologist.13-S2-4

[3] JOENSUU H, ERIKSSON M, HALL KS, HARTMANN JT, PINK D et al. Risk factors for gastrointestinal stromal tumor recurrence in patients treated with adjuvant imatinib. Cancer 2014; 120: 2325-2333. https://doi.org/10.1002/cncr.28669

[4] JOENSUU H, VEHTARI A, RIIHIMAKI J, NISHIDA T, STEIGEN SE et al. Risk of recurrence of gastrointestinal stromal tumour after surgery: an analysis of pooled population based cohorts. Lancet Oncol 2012; 13: 265-274. https://doi. org/10.1016/S1470-2045(11)70299-6

[5] JOENSUU H, ROBETTS PJ, SARLOMO-RIKALA M, ANDERSSON LC, TERVAHARTIALA $P$ et al. Effect of the tyrosine kinase inhibitor STI571 in a patient with a metastatic gastrointestinal stromal tumor. N Engl J Med 2001; 344: 1052-1056. https://doi.org/10.1056/ NEJM200104053441404

[6] LOUGHREY MB, MITCHELL C, MANN GB. MICHAL M, WARIN PM. Gastrointestinal stromal tumour treated with neoadjuvant imatinib. J Clin Pathol 2005; 58: 779-781. https:// doi.org/10.1136/jcp.2004.023226

[7] DENNIS KL, DAMJANOV I. Evaluating and reporting gastrointestinal stromal tumors after imatinib mesylate treatment. Open J Pathol 2009; 3: 53-57. https:// doi.org/10.2174/1874375700903020053

[8] LIEGL-ATZWANGER B, FLETCHER JA, FLETCHER CD. Gastrointestinal stromal tumors. Virchows Arch 2010; 456: 111-127. https://doi.org/10.1007/s00428-010-0891-y

[9] YANAGAWA S, TANABE K, SUZUKI T, TOKUMOTO N, AIRIHOR $\mathrm{K}$ et al. A large gastrointestinal stromal tumour that was succesfully resected after neoadjuvant imatinib treatment: A case report. World J Surg Oncol 2014; 12: 47-50. https:// doi.org/10.1186/1477-7819-12-47

[10] JOENSUU H, RUTKOWSKI P, NISHIDA T, STEIGEN SE, BRABEC $P$ et al. KIT and PDGFRA mutations and the risk of GI stromal tumor recurrence. J Clin Oncol 2015; 33: 634-642. https://doi.org/10.1200/JCO.2014.57.4970

[11] BENJAMIN RS, BLANKE CD, BLAY JY, BONVALOT S, EISENBERG B. Management of gastrointestinal stromal tumors in the Imatinib era: Selected case studies. The Oncologist 2006; 11: 9-20. https://doi.org/10.1634/theoncologist.11-1-9

[12] ANTONESCU CR, BESMER P, GUO T, ARKUN K, HOM $\mathrm{G}$. Acquired resistance to imatinib in gastrointestinal stromal tumor occurs through secondary gene mutation. Clin Cancer Res 2005; 11: 4182-4190. https://doi.org/10.1158/1078-0432. CCR-04-2245

[13] FLETCHER CD, BERMAN JJ, CORLESS C, GORSTEIN F, LASOTA J et al. Diagnosis of gastrointestinal stromal tumors: A consensus approach. Hum Pathol 2002; 33: 459-465. https:// doi.org/10.1053/hupa.2002.123545

[14] MIETTINEN M, LASOTA J. Gastrointestinal stromal tumors: review on morphology, molecular pathology, prognosis, and differential diagnosis. Arch Pathol Lab Med 2006; 130: 1466-1478.

[15] BOSMAN FT, CARNEIRO F, HRUBAN RH, THEISE ND. (Eds.) WHO Classification of Tumours of the Digestive System. Lyon: IARC, 2010, 417 pp. ISBN 9789283224327.

[16] MINARIK G, PLANK L, LASABOVA Z, SZEMES T, BURJANIVOVA T et al. Spectrum of mutations in gastrointestinal stromal tumor patients - a population-based study from Slovakia. APMIS 2013; 121:539-548. https://doi.org/10.1111/ apm.12019

[17] BENJAMIN RS, CHOI H, MACAPINLAC HA, BURGESS MA, PATEL SS et al. We should desists using RECIST, at least in GIST. J Clin Oncol 2007; 25: 1760-1764. https://doi. org/10.1200/JCO.2006.07.3411

[18] KALKMANN J, ZEILE M, ANTOCH G, BERGER F, DIEDERICH S et al. Consensus report on the radiological management of patients with gastrointestinal stromal tumours (GIST): recommendation of the German GIST Imaging Working Group. Cancer Imaging 2012; 12: 126-135. https://doi. org/10.1102/1470-7330.2012.0013

[19] GONG J, KANG W, ZHU J, XU J. CT and MR imaging of gastrointestinal stromal tumor of stomach: a pictorial review. Quant Imaging Med Surg 2012; 2: 274-279.

[20] LUCAS DR, KSHIRSAGAR MP, BIERMAN JS, HAMRE MR, THOMAS DG et al. Histologic alterations from neodajuvant chemotherapy in high-grade extremity soft tissue sarcoma: clinicopathological correlation. Oncologist 2008; 13: 451-458. https://doi.org/10.1634/theoncologist.2007-0220 
[21] LINTON KM, TAYLOR MB, RADFORD JA. Response evaluation in gastrointestinal stromal tumours treated with imatinib: misdiagnosis of disease progression on CT due to cystic change in liver metastases. Br J Radiol 2006; 79: e40-44. https://doi.org/10.1259/bjr/62872118

[22] ANTONESCU CR. Targeted therapy of cancer: New roles for pathologists in identifying GISTs and other sarcomas. Mod Pathol 2008; (Suppl 2): S31-36.
[23] BAUER S, HARTMANN JT, deWIT M, LANG H, GRABELLUS $F$ et al. Resection of residual disease in patients with metastatic gastrointestinal stromal tumor responding to treatment with imatinib. Int J Cancer 2005; 117: 316-325. https:// doi.org/10.1002/ijc.21164

[24] LIEGL B, KEPTEN I, LE C, ZHU M, DEMETRI GD et al. Heterogeneity of kinase inhibitor resistance mechanisms in GIST. J Pathol. 2008; 216: 64-74. https://doi.org/10.1002/path.2382 